\title{
(D)
}

\section{The Effects of Trunk Control on Respiratory Muscle Strength and Activities of Daily Living in Children with Cerebral Palsy}

\author{
Umut Apaydin ${ }^{1,{ }^{*}}$, Zeynep Aribas ${ }^{1}$, Erkan Erol ${ }^{1}$, Yasemin Aydin ${ }^{1}$, Murat Fatih Kocyigit ${ }^{1}$ and Bulent \\ Elbasan ${ }^{1}$ \\ ${ }^{1}$ Department of Physiotherapy and Rehabilitation, Faculty of Health Sciences, Gazi University, Ankara, Turkey \\ "Corresponding author: Department of Physiotherapy and Rehabilitation, Faculty of Health Sciences, Gazi University, Emniyet Mah. Muammer Yaşar Bostancı Cad. No: 16 \\ Beșevler, Ankara, Turkey. Tel: +90-3122162643, Fax: +90-3122162636, Email: fzt.umut28@gmail.com
}

Received 2018 April 24; Revised 2018 September 14; Accepted 2018 September 15.

\begin{abstract}
There is not enough evidence regarding the effects of trunk control on respiratory muscle strength and activities of daily living (ADL) in children with cerebral palsy (CP). This study was planned to investigate the effects of trunk control on respiratory muscle strength and ADL in children with spastic CP. A total of 34 children with spastic CP (M \pm SD age, $9.3 \pm 2.4$ years) and 30 typical peers ( $M \pm$ SD age, $9.7 \pm 2.1$ years) were included in the study. Trunk control was evaluated by the Trunk Control Measurement Scale (TCMS), ADL was evaluated by Pediatric Evaluation of Disability Inventory (PEDI), and respiratory muscle strength was evaluated by mouth pressure meter. The trunk control, respiratory muscle strength, and ADL of the children with spastic CP were significantly lower than their typically peers $(\mathrm{P}<0.001$, respectively). There were statistically significant correlations between the trunk control and maximum inspiratory pressure (MIP) $(r=0.503, P<0.05)$, maximum expiratory pressure $(\mathrm{MEP})(\mathrm{r}=0.465, \mathrm{P}<0.05)$, mobility subscale of Functional Skills Scale of PEDI (FSS-MS) $(r=0.570, P<0.001)$, self-care subscale of Caregiver Assistance Scale of PEDI (CAS-SS) $(\mathrm{r}=0.431, \mathrm{P}<0.05)$, mobility subscale of Caregiver Assistance Scale of PEDI (CAS-MS) $(\mathrm{r}=.607, \mathrm{P}<0.001)$, and Gross Motor Functional Classification System (GMFCS) $(r=-0.522, \mathrm{P}<0.05)$ in children with spastic CP. In addition, MIP, MEP, PEDI FSS-MS, CAS-SS, and CAS-MS were independently predictors of trunk control in children with spastic CP. The results of this study demonstrate that trunk control in children with spastic CP is correlated positively to MIP, MEP values and their daily living activities and we suggest this should be taken into account when planning an intervention to improve the ability of daily living function for children with spastic CP.
\end{abstract}

Keywords: Cerebral Palsy, Trunk Control, Respiratory Muscle Strength, Activities of Daily Living

\section{Background}

Cerebral palsy (CP) can be defined as a group of disorders of movement and posture causing activity limitation that are attributed to non-progressive deficits in the immature brain (1). The motor disorders in CP are often accompanied by the deficits in cognition, communication, perception, respiration, behavior, and sensory system $(2,3)$.

Children with $\mathrm{CP}$ have many primary motor impairments such as the lack of selectivity, mobility, muscle weakness, abnormal muscle tone, impaired coordination between the agonist-antagonist muscles, and lack of postural control. These motor impairments also lead to secondary problems such as contractures and bony deformities further in life. Whether primary or secondary, all these problems may reduce independence in the activities of daily living (ADL) in children with CP with different severity (4).

Postural control is defined as ensuring proper posi- tioning of the body in space as well as maintaining body alignment and stability by controlling the center of gravity in the base of support (5). Dysfunction in postural control is a key problem in children with CP. Trunk control is one of the primary aspects providing postural control. The trunk serves as the center of our body, which acts as a stable base of support during upper and lower limb activities, provides regulation of balance reactions and successful execution of functional activities (6) and has an active role in activities such as reaching and walking (7). The lack of control in the trunk affects functional mobility and balance as well as limiting participation in $\operatorname{ADL}(4,8)$ in children with CP.

Pulmonary dysfunction is also a common problem due to the motor impairment of the respiratory muscles in children with $\mathrm{CP}$. These children may cope with poor coordination patterns, low tidal volume, and decreased cardiopulmonary capacity in respiratory muscles. Accord- 
ingly, parenchymal lung pathologies, such as diffuse small atelectasis and decreased lung tension may also be seen. As a result, these pathologies can also affect motor development and performance of ADL in children with $\mathrm{CP}$ (9).

From the biomechanical perspective, respiratory muscles especially diaphragm, also serves for postural stability and control (10). Several studies concluded that there is a relationship between the activity of the diaphragm and intercostal muscles and both respiratory and postural functions (10-12). However, there is no information regarding the relationship between trunk control and respiratory muscle strength in children with spastic CP. This study was planned to investigate the effects of trunk control on ADL and respiratory muscle strength in children with CP in level 1 or 2 according to the Gross Motor Function Classification System (GMFCS).

\section{Methods}

This study had $90 \%$ power and 5\% standard error. A total of 21 children with hemiplegia, 13 children with diplegia between the ages 6 - 15 years, and 30 typical peers between the ages 7 - 14 years were included in this study. The inclusion criteria for the children with CP were; to be at the level of 1 or 2 in GMFCS, having neither orthopedic surgery nor Botulinum Toxin-A injection in the last 6 months, having no cooperation problems and scoliosis during postural evaluation. Demographic data of all children were recorded and the GMFCS levels were determined by a five-year experienced pediatric physiotherapist.

Trunk Control Measurement Scale (TCMS) was used to assess the trunk control of the participants. TCMS measures the state of balance on the support surface and the ability to actively move body parts during functional activities, which are the two components of trunk control. TCMS consists of 15 items in total that are scored on 2,3, or 4 point ordinal scale and administered bilaterally in case of clinical relevance. The total TCMS score ranges from 0 to 58. A high score on this scale represents a better performance (13). It has been shown that TCMS is a valid and reliable scale for assessing trunk control in children with spastic CP(13).

The ADL of the children were assessed by the Pediatric Evaluation of Disability Inventory (PEDI), which is a clinical measurement developed by Haley and is used to evaluate the change in the functional skills, functional abilities, and performance of children with disabilities (14). PEDI consists of two sections as Functional Skills Scale and Caregiver Assistance Scale. The Functional Skills Scale, is divided into three subscales: Self-care, mobility, and social function, which consists of 197 items in total, is scored as unable as "0", and capable as " 1 ". The Caregiver Assistance
Scale, is also divided into three subscales: self-care, mobility, and social function, which consist of 20 items in total. Each item in this section is scored between " 0 " and 5". A score of 5 indicates that the child is completely independent, while a score of 0 indicates that the child is completely dependent on the caregiver. It has been shown that PEDI is a valid and reliable inventory for children in Turkey (15).

Respiratory functions were assessed by measuring the respiratory muscle strength. These measurements were done by using the respiratory pressure meter (Micro Medical Micro RPM, UK) and performed according to American Thoracic Society/European Respiratory Society criteria (16). Maximal Inspiratory Pressure (MIP) was measured in the residual volume after maximal expiration, while Maximal Expiratory Pressure (MEP) was measured in total lung capacity after maximum inspiration (17). The measurements were done in a sitting position by a physiotherapist with a 10 year experience in the field of pediatric pulmonary rehabilitation. She was blinded to the TCMS and PEDI scores of the children. The children were encouraged verbally by the physiotherapist and nose clips were used during the process. Each measurement was repeated three times and the highest score was recorded (within \%5) and expressed in $\mathrm{cm} \mathrm{H}_{2} \mathrm{O}$.

All evaluations were done in the morning at Gazi University, Department of Physiotherapy and Rehabilitation, Pediatric Rehabilitation Unit. Each child was first assessed for respiratory muscle strength and then trunk control. A total break of 10 minutes was given for all children between the evaluations to prevent fatigue; all evaluations took an average of 90 minutes. If the child was unable to complete the assessment in one session they are recalled on the next day to finalize the assessment.

The information regarding the study was sent to the regular schools for obtaining the control group. The volunteers who agreed on participating in the study were evaluated at the Pediatric Rehabilitation unit of the Physiotherapy and Rehabilitation Department at Gazi University. The study was approved by the Ethics Committee of the Kecioren Training and Research Hospital, Ankara, Turkey (protocol no: 2012-KAEK-15/1572) and performed in accordance with the Declaration of Helsinki.

\subsection{Statistical Analysis}

IBM® SPSS (C) 22 software was used for the statistical analysis of the study. Visual (histogram and probability plots) and analytical methods (Shapiro Wilk test) were used to determine whether the data were normally distributed. The Independent Samples $t$-test was used to compare two independent groups when the normal distribution conditions were met, and the Mann-Whitney U test was used to compare two independent groups when 
normal distribution conditions were not met. Correlation coefficients and statistical significance for normal distributed numerical variables were calculated by Pearson Correlation test. Correlation coefficients and statistical significance were evaluated by Spearman Correlation test for inter-variable relationships, at least one of which was normally non-distributed or ordinal. In addition, the effects of trunk control on respiratory muscle strength and ADLs were determined by simple linear regression analysis. The type 1 error level was accepted as $5 \%$ for statistical significance.

\section{Results}

A total of 36 children with CP and 32 typical peers were included in the study. Two children with CP were excluded due to not meeting the inclusion criteria (1 child with CP had a Botox injection one week before the evaluation, 1 child with $\mathrm{CP}$ and 2 children in the control group could not cooperate during the respiratory muscle strength testing). Therefore, this study was completed with 34 children with CP (21 hemiparetic, 13 diparetic) and 30 typical peers. Demographic characteristics of both groups were shown in Table 1. There was no difference between the groups in terms of gender, age, height, weight, and body mass in$\operatorname{dex}(\mathrm{BMI})(\mathrm{P}>0.05)$. The trunk control, respiratory muscle strength, and ADLs of the children with CP were significantly lower than their typical peers $(\mathrm{P}<0.001)$ (Table 2$)$.

The total score of TCMS was positively correlated with MIP ( $\mathrm{r}=0.503, \mathrm{P}<0.05)$, MEP $(\mathrm{r}=0.465, \mathrm{P}<0.05)$, mobility subscale of Functional Skills Scale of PEDI (FSS-MS) $(\mathrm{r}=0.570, \mathrm{P}<0.001)$, self-care subscale of Caregiver Assistance Scale of PEDI (CAS-SS) ( $\mathrm{r}=0.431, \mathrm{P}<0.05)$, mobility subscale of Caregiver Assistance Scale of PEDI (CAS-MS) ( $\mathrm{r}=$ $0.607, \mathrm{P}<0.001)$, and negatively correlated with GMFCS ( $\mathrm{r}$ $=-0.522, \mathrm{P}<0.05)$. On the other hand, the scores of MIP and MEP were significantly correlated with self-care subscale of Functional Skills Scale of PEDI (FSS-SS) $(\mathrm{r}=0.617, \mathrm{P}<0.001$; $\mathrm{r}=0.584, \mathrm{P}<0.001$, respectively) and CAS-SS of PEDI $(\mathrm{r}=$ $0.711, \mathrm{P}<0.001 ; \mathrm{r}=0.520, \mathrm{P}<0.05$, respectively) in children with CP. There was also a positive correlation between trunk control and MIP $(\mathrm{r}=0.469, \mathrm{P}<0.05), \mathrm{MEP}(\mathrm{r}=0.355$, $\mathrm{P}<0.05)$, PEDI FSS-SS $(\mathrm{r}=0.409, \mathrm{P}<0.05)$, and PEDI CAS-SS $(\mathrm{r}=0.414, \mathrm{P}<0.05)$ in their typical peers (Table 3 ).

It was determined that, the trunk control affects the MIP, MEP, PEDI FSS-MS, PEDI CAS-SS, and PEDI CAS-MS scores in children with spastic CP (Table 4).

\section{Discussion}

There was not enough evidence stating the effect of trunk control on respiratory muscle strength in children with spastic CP. A number of studies have examined the relationship between the respiratory functions, sitting postures and sitting frames of children with CP $(18,19)$. So, this is the first study examining the relationship of trunk control with respiratory muscle strength in children with spastic $C P$, who have mild involvement. It was seen that, trunk control affects the MIP, MEP and ADLs in children with spastic $\mathrm{CP}$ at the level of 1 and 2 according to the GMFCS.

Although, the study showed that the children with CP had similar demographic characteristics with their control peers, it was observed that the TCMS scores were lower in children with $\mathrm{CP}$, which is in line with the findings of Heyrman et al. (13, 20). Trunk may affect the mobility, which is an important parameter for ADLs. As Saether et al. and Heyrman et al. reported trunk control was associated with trunk movements during walking, and the trunk took an active role during walking $(4,8)$, which shows that the low TCMS scores were correlated with poor performance during walking. It was found that the increase in the TCMS score resulted in an increase in all sub-parameter scores in PEDI except for the self-care subscale of FSS section in the present study. The absence of correlation between the trunk control and the self-care subscale of FSS in PEDI may be due to the lack of the relationship between the trunk and the upper limb functions. Since majority of the children involved in the study were children with hemiplegia, even though the trunk control was good, their fine motor skills during these activities may have been limited. Similarly, the protective attitudes of parents giving no chance to try the activities of daily living such as washing the body and face may also contribute to this problem. Accordingly, children with better trunk control had better mobility function and need less caregiver assistance in the activities of daily living.

Children with CP may have problems with respiratory muscle strength due to postural and accompanying secondary musculoskeletal problems. Studies have shown that respiratory muscle strength weakness can be seen with different severity in children with CP. In this study, MIP and MEP outcomes of the children with spastic CP were found to be lower compared to their typical peers. Wang et al. also reported that respiratory muscle strength was lower in children with CP when compared to the controls (21). Kwon and Lee have reported that respiratory muscle strength and pulmonary function of both spastic hemiparetic and diparetic children were lower compared to those of children with typical development (9). Similar results of this paper and other studies in the literature suggest that respiratory muscle strength problems in children with CP should not be ignored and should be strengthened in rehabilitation programs.

Nwaobi and Smith have examined the effects of sitting 


\begin{tabular}{|c|c|c|c|}
\hline Demographic Characteristics & Children with Cerebral Palsy, N: 34 & Control Group, $\mathbf{N}: 30$ & $\mathbf{P}$ \\
\hline Gender & & & $0.975^{\mathrm{a}}$ \\
\hline Female & $18(52.9)$ & $16(53.3)$ & \\
\hline Male & $16(47.1)$ & $14(46.7)$ & \\
\hline Age, $y$ & & & $0.472^{\mathrm{b}}$ \\
\hline Mean \pm SD & $9.3 \pm 2.4$ & $9.7 \pm 2.1$ & \\
\hline Range (min - max) & $6-15$ & 7-14 & \\
\hline Height, cm & $132.5 \pm 15.6$ & $138.6 \pm 13.2$ & $0.103^{b}$ \\
\hline Weight, kg & $30(24-36)$ & $28.5(25-45)$ & $0.721^{\mathrm{C}}$ \\
\hline Body mass index, $\mathrm{kg} / \mathrm{m}^{2}$ & $17.4 \pm 3.1$ & $16.9 \pm 3.4$ & $0.537^{\mathrm{b}}$ \\
\hline \multicolumn{4}{|l|}{ Topographic classification } \\
\hline Hemiparetic & $21(61.8)$ & & \\
\hline Diparetic & $13(38.2)$ & & \\
\hline \multicolumn{4}{|l|}{ GMFCS } \\
\hline Level 1 & $21(61.8)$ & & \\
\hline Level 2 & $13(38.2)$ & & \\
\hline
\end{tabular}

Abbreviations: SD, standard deviation; min, minimum; max, maximum; GMFCS, Gross Motor Functional Classification System.

${ }^{a}$ Chi-square test.

${ }^{\mathrm{b}}$ Independent Samples $t$-test.

${ }^{\mathrm{c}}$ Mann-Whitney U test.

\begin{tabular}{|c|c|c|c|}
\hline & Children with CP, N: 34 & Control Group, N: 30 & $\mathbf{P}$ \\
\hline TCMS-SSB score & $20(19-20)$ & $20(20-20)$ & $0.003^{\mathrm{a}}$ \\
\hline TCMS-SMC score & $19.2 \pm 4.1$ & $26 \pm 1.8$ & $<0.001^{\mathrm{b}}$ \\
\hline TCMS-DR score & $6.2 \pm 2.3$ & $9.8 \pm 0.5$ & $<0.001^{\mathrm{b}}$ \\
\hline Total TCMS score & $44.5 \pm 7.4$ & $55.7 \pm 1.8$ & $<0.001^{\mathrm{b}}$ \\
\hline MIP & $67.5 \pm 28.9$ & $98.9 \pm 26.7$ & $<0.001^{\mathrm{b}}$ \\
\hline MEP & $72.5 \pm 25.8$ & $108.4 \pm 26.1$ & $<0.001^{\mathrm{b}}$ \\
\hline PEDI (FSS-SS) & $67(62-68)$ & $73(73-73)$ & $<0.001^{\mathrm{a}}$ \\
\hline PEDI (FSS-MS) & $56(49-58)$ & $59(59-59)$ & $<0.001^{\mathrm{a}}$ \\
\hline PEDI (CAS-SS) & $34(31-38)$ & $40(40-40)$ & $<0.001^{\mathrm{a}}$ \\
\hline PEDI (CAS-MS) & $34(30-35)$ & $35(35-35)$ & $<0.001^{\mathrm{a}}$ \\
\hline
\end{tabular}

Abbreviations: TCMS, Trunk Control Measurement Scale; SSB, static sitting balance; SMC, selective movement control; DR, dynamic reaching; MIP, maximal inspiratory pressure; MEP, maximal expiratory pressure; PEDI, Pediatric Evaluation of Disability Inventory; FSS, Functional Skills Scale; CAS, Caregiver Assistance Scale; SS, self-Ccare subscale; MS, mobility subscale; $\mathrm{CP}$, cerebral palsy.

${ }^{a}$ Mann-Whitney U test.

${ }^{\mathrm{b}}$ Independent Samples $t$-test.

in an adaptive and non-adaptive chair, on respiratory functions in eight spastic non-ambulatory children with CP between the ages 5 and 12 years (19). It was concluded that the adaptive sitting chair provided better upright posture and alignment, therefore, they reported that the vital capacity (VC), forced expiratory volume in 1 second (FEV1), and expiratory time parameters of children with $\mathrm{CP}$, were better.
Therefore, the adaptive siting system increase the physical capacity of the thorax and abdomen, leads to a better control of the respiratory muscles, and minimize the airway obstruction with the provision of postural straightness.

In another study, Barks and Davenport examined the effect of wheelchair components on respiratory function in children with CP (18). They stated that the upper extrem- 


\begin{tabular}{|c|c|c|c|c|c|c|}
\hline & \multicolumn{3}{|c|}{ Children with CP, N: 34} & \multicolumn{3}{|c|}{ Control Group, N: 30} \\
\hline & Total TCMS score & MIP & MEP & Total TCMS score & MIP & MEP \\
\hline Age, $y$ & $0.055^{\mathrm{b}}$ & $0.538^{* b}$ & $0.256^{\mathrm{b}}$ & $0.447^{* \mathrm{~b}}$ & $0.716^{* * \mathrm{~b}}$ & $0.723^{* * \mathrm{~b}}$ \\
\hline BMI, $\mathrm{kg} / \mathrm{m}^{2}$ & $-0.242^{\mathrm{b}}$ & $0.075^{\mathrm{b}}$ & $0.032^{\mathrm{b}}$ & $0.386^{* c}$ & $0.621^{* * \mathrm{~b}}$ & $0.674^{* * \mathrm{~b}}$ \\
\hline MIP & $0.503^{* \mathrm{~b}}$ & - & - & $0.469^{* c}$ & - & - \\
\hline MEP & $0.465^{* \mathrm{~b}}$ & - & - & $0.355^{* c}$ & - & - \\
\hline PEDI (FSS-SS) & $0.288^{\mathrm{c}}$ & $0.617^{* * \mathrm{c}}$ & $0.584^{* * \mathrm{c}}$ & $0.409^{* c}$ & $0.529^{* c}$ & $0.595^{* \mathrm{c}}$ \\
\hline PEDI (FSS-MS) & $0.570^{* * \mathrm{c}}$ & $0.135^{c}$ & $0.228^{\mathrm{C}}$ & - & - & - \\
\hline PEDI (CAS-SS) & $0.431^{* \mathrm{C}}$ & $0.711^{* * c}$ & $0.520^{* \mathrm{c}}$ & $0.414^{* \mathrm{c}}$ & $0.531^{* c}$ & $0.593^{* \mathrm{c}}$ \\
\hline PEDI (CAS-MS) & $0.607^{* * c}$ & $0.214^{\mathrm{c}}$ & $0.255^{\mathrm{c}}$ & - & - & - \\
\hline GMFCS & $-0.522^{* \mathrm{C}}$ & $-0.069^{c}$ & $-0179^{c}$ & - & - & - \\
\hline
\end{tabular}

Abbreviations: BMI, body mass index; TCMS, Trunk Control Measurement Scale; MIP, maximum inspiratory pressure; MEP, maximum expiratory pressure; PEDI, Pediatric Evaluation of Disability Inventory; FSS, Functional Skills Scale; CAS, Caregiver Assistance Scale; SS, self-care subscale; MS, mobility subscale; GMFCS, Gross Motor Functional Classification System; CP, cerebral palsy.

a $*$ P $<0.05 ;{ }^{* *}, \mathrm{P}<0.001$.

${ }^{\mathrm{b}}$ Pearson Correlation Test.

${ }^{c}$ Spearman Correlation Test.

\begin{tabular}{lccccc}
\hline $\begin{array}{l}\text { Table 4. The Effect of Trunk Control on Other Parameters in Children with CP } \\
\begin{array}{l}\text { Dependent } \\
\text { Variables }\end{array}\end{array}$ & Total TCMS Score & $\mathbf{R}^{\mathbf{2}}$ & $\mathbf{P}$ & \multicolumn{2}{c}{ Confidence Interval } \\
\hline MIP & 0.094 & 0.135 & 0.033 & Lower & Upper \\
\hline MEP & 0.100 & 0.123 & 0.042 & 0.008 & 0.180 \\
\hline PEDI (FSS-SS) & 0.372 & 0.070 & 0.131 & 0.004 & 0.197 \\
PEDI (FSS-MS) & 0.600 & 0.400 & 0.001 & -0.116 & 0.860 \\
\hline PEDI (CAS-SS) & 0.599 & 0.174 & 0.014 & 0.335 & 0.865 \\
PEDI (CAS-MS) & 1.033 & 0.434 & $<0.001$ & 0.130 & 1.068 \\
\hline
\end{tabular}

Abbreviations: TCMS: Trunk Control Measurement Scale, MIP: maximal inspiratory pressure, MEP: maximal expiratory pressure, PEDI: Pediatric Evaluation Of Disability Inventory, FSS: Functional Skills Scale, CAS: Caregiver Assistance Scale, SS: self-care subscale, MS: mobility subscale. Simple linear regression analysis was used.

${ }^{a}$ Simple linear regression analysis was used.

ity support or side trunk supports on the wheelchair provided a reduction in airway resistance and better ventilation of the lungs by positioning the trunk in a straight position. These studies included non-ambulatory children with CP who were severely involved. On the other hand, we found that the trunk control was positively associated with the MIP and MEP score in children with spastic CP in better functional levels according to GMFCS. In particular, an increase in the TCMS score by one point lead to an average increase of $0.094 \mathrm{~cm} \mathrm{H}_{2} \mathrm{O}$ in the MIP score and $0.1 \mathrm{~cm}$ $\mathrm{H}_{2} \mathrm{O}$ in the MEP score. This result may be related to the increased trunk control as well as the reduced airway resistance and abdominal load, increased thoracic expansion, and better functioning of the inspiratory and expiratory muscles, which is parallel to the findings in the literature $(18,19)$.

Deficiency in respiratory functions in children with CP can also affect ADLs. To the best of our knowledge, there is only one study investigating the relationship between the respiratory functions and ADLs in children with CP(21). In the present study, MIP and MEP scores were correlated with the FSS-SS and the CAS-SS of PEDI. These results seem to be similar to the study done by Wang et al. (21), and it is thought that this research is important in showing that the caregivers also agree with this result. There was no correlation between the respiratory muscle strength and the FSS-MS, and the CAS-MS of PEDI. Assessment of other pulmonary functions such as respiratory muscle endurance as well as respiratory muscle strength may also provide important information regarding the mobility level of the children. In addition, since the study group consisted of children with better GMFCS levels and MIP scores, this may also affect the mobility functions of the children. These results show that children with better respiratory muscle 
strength perform better in self-care and need less caregiver assistance in their daily living activities. Therefore, the inclusion of the respiratory physiotherapy programs in the early rehabilitation approaches may contribute to the level of independence in the ADLs. The trunk control was assessed with TCMS, which is costless and can be applied easily in all clinics. The lack of evaluation tools such as 3D gait analysis systems, including the trunk or electromyographic systems that directly assess muscles instead of TCMS, can be accepted as a limitation of this study. In addition, the non-evaluation of children's aerobic capacities in this research may be considered as a limitation. We included the children with CP with better GMFCS levels in the present study. Future studies should investigate the relationship between the trunk control and respiratory muscle strength in GMFCS levels III-V children with CP whose functional limitations are higher.

In conclusion, trunk control may affect many parameters even in children with CP who's GMFCS levels are 1 and 2. These results indicate that more attention should be paid to the trunk control in physiotherapy and rehabilitation programs of children with CP. In addition, respiratory muscle strength, which has an important correlation with the trunk control and ADLs, should not be neglected in the rehabilitation programs.

\section{Footnotes}

Authors' Contribution: All authors participated in the design of the study. Bulent Elbasan was the director of the study and was responsible for analysis, interpretation and final draft preparation. Umut Apaydin performed data collection, analysis, interpretation and prepared the manuscript. Murat Fatih Kocyigit and Erkan Erol performed data collection and analysis. Yasemin Aydin performed data collection. A final draft was prepared incorporating suggestions from Zeynep Aribas. All authors declare that they have had access and take responsibility for the integrity of the data and the accuracy of the data analysis.

Ethical Considerations: The study was approved by the Ethics Committee of the Kecioren Training and Research Hospital, Ankara, Turkey (protocol no: 2012-KAEK15/1572) and performed in accordance with the Declaration of Helsinki.

\section{References}

1. Jones MW, Morgan E, Shelton JE, Thorogood C. Cerebral palsy: Introduction and diagnosis (part I). J Pediatr Health Care. 2007;21(3):146-52. doi:10.1016/j.pedhc.2006.06.007. [PubMed:17478303].

2. Bax M, Goldstein M, Rosenbaum P, Leviton A, Paneth N, Dan $\mathrm{B}$, et al. Proposed definition and classification of cerebral palsy, April 2005. Dev Med Child Neurol. 2005;47(8):571-6. doi: 10.1017/S001216220500112X. [PubMed: 16108461].
3. Rosenbaum P, Paneth N, Leviton A, Goldstein M, Bax M, Damiano D, et al. A report: The definition and classification of cerebral palsy April 2006. Dev Med Child Neurol Suppl. 2007;109:8-14. doi: 10.1111/j.14698749.2007.tb12610.x. [PubMed: 17370477].

4. Heyrman L, Feys H, Molenaers G, Jaspers E, Monari D, Nieuwenhuys A, et al. Altered trunk movements during gait in children with spastic diplegia: Compensatory or underlying trunk control deficit? Res Dev Disabil. 2014;35(9):2044-52. doi: 10.1016/j.ridd.2014.04.031. [PubMed: 24864057].

5. Pavao SL, Nunes GS, Santos AN, Rocha NA. Relationship between static postural control and the level of functional abilities in children with cerebral palsy. Braz J Phys Ther. 2014;18(4):300-7. doi: 10.1590/bjptrbf.2014.0056. [PubMed: 25054383]. [PubMed Central: PMC4183259].

6. van der Heide JC, Begeer C, Fock JM, Otten B, Stremmelaar E, van Eykern LA, et al. Postural control during reaching in preterm children with cerebral palsy. Dev Med Child Neurol.2004;46(4):253-66. doi: 10.1017/S0012162204000416. [PubMed: 15077703].

7. Mayston MJ. People with cerebral palsy: Effects of and perspectives for therapy. Neural Plast. 2001;8(1-2):51-69. doi: 10.1155/NP.2001.51. [PubMed: 11530888]. [PubMed Central: PMC2565384].

8. Saether R, Helbostad JL, Adde L, Braendvik S, Lydersen S, Vik T. The relationship between trunk control in sitting and during gait in children and adolescents with cerebral palsy. Dev Med Child Neurol. 2015;57(4):344-50. doi: 10.1111/dmcn.12628. [PubMed: 25412837].

9. Kwon YH, Lee HY. Differences of respiratory function according to level of the gross motor function classification system in children with cerebral palsy. J Phys Ther Sci. 2014;26(3):389-91. doi: 10.1589/jpts.26.389. [PubMed: 24707090]. [PubMed Central: PMC3976009].

10. Gonzalez-Alvarez FJ, Valenza MC, Torres-Sanchez I, Cabrera-Martos I, Rodriguez-Torres J, Castellote-Caballero Y. Effects of diaphragm stretching on posterior chain muscle kinematics and rib cage and abdominal excursion: A randomized controlled trial. Braz J Phys Ther. 2016;20(5):405-11. doi: 10.1590/bjpt-rbf.2014.0169. [PubMed: 27333481]. [PubMed Central: PMC5123258].

11. Hodges PW, Gandevia SC. Changes in intra-abdominal pressure during postural and respiratory activation of the human diaphragm. J Appl Physiol (1985). 2000;89(3):967-76. doi: 10.1152/jappl.2000.89.3.967. [PubMed: 10956340].

12. De Troyer A, Kirkwood PA, Wilson TA. Respiratory action of the in tercostal muscles. Physiol Rev. 2005;85(2):717-56. doi: 10.1152/physrev.00007.2004. [PubMed: 15788709].

13. Heyrman L, Molenaers G, Desloovere K, Verheyden G, De Cat J, Monbaliu E, et al. A clinical tool to measure trunk control in children with cerebral palsy: The Trunk Control Measurement Scale. Res Dev Disabil. 2011;32(6):2624-35. doi:10.1016/j.ridd.2011.06.012. [PubMed: 21757321].

14. Haley SM, Coster WJ, Ludlow LH, Haltiwanger JT; The Psychological Corporation; New England Medical Center Hospital PEDI Research Group, et al. Pediatric evaluation of disability inventory (PEDI): Development, standardization and administration manual. Boston, MA: Psychological Corporation; 1992.

15. Erkin G, Aybay C, Kurt M, Keles I, Cakci A, Ozel S. The assessment of functional status in Turkish children with cerebral palsy (a preliminary study). Child Care Health Dev. 2005;31(6):719-25. doi: 10.1111/j.13652214.2005.00565.x. [PubMed:16207230].

16. American Thoracic Society/European Respiratory S. ATS/ERS Statement on respiratory muscle testing. Am J Respir Crit Care Med. 2002;166(4):518-624. doi: 10.1164/rccm.166.4.518. [PubMed: 12186831].

17. Domenech-Clar R, Lopez-Andreu JA, Compte-Torrero L, De DiegoDamia A, Macian-Gisbert V, Perpina-Tordera M, et al. Maximal static respiratory pressures in children and adolescents. Pediatr Pulmonol. 2003;35(2):126-32. doi:10.1002/ppul.10217. [PubMed:12526074].

18. Barks L, Davenport P. Wheelchair components and pulmonary function in children with cerebral palsy. Assist Technol. 2012;24(2):78-86. doi: 10.1080/10400435.2012.659793. [PubMed: 22876730]. 
19. Nwaobi OM, Smith PD. Effect of adaptive seating on pulmonary function of children with cerebral palsy. Dev Med Child Neurol. 1986;28(3):351-4. doi: 10.1111/j.1469-8749.1986.tb03883.x. [PubMed: 3721078].

20. Heyrman L, Desloovere K, Molenaers G, Verheyden G, Klingels K, Monbaliu E, et al. Clinical characteristics of impaired trunk control in chil- dren with spastic cerebral palsy. Res Dev Disabil. 2013;34(1):327-34. doi: 10.1016/j.ridd.2012.08.015. [PubMed: 23000634].

21. Wang HY, Chen CC, Hsiao SF. Relationships between respiratory muscle strength and daily living function in children with cerebral palsy. Res Dev Disabil. 2012;33(4):1176-82. doi: 10.1016/j.ridd.2012.02.004. [PubMed: 22502843]. 